\title{
GRUPO DE GINÁSTICA GERAL DA UNIVERSIDADE PRESBITERIANA MACKENZIE: CARACTERÍSTICAS FÍSICAS E MOTIVOS DE ADESÃO
}

\author{
Olavo Dias de Souza Junior, Universidade Presbiteriana Mackenzie, São Paulo - Brasil \\ Érico Chagas Caperuto, Universidade Presbiteriana Mackenzie, São Paulo - Brasil \\ Vinicius Barroso Hirota, Universidade Presbiteriana Mackenzie, São Paulo - Brasil \\ André dos Santos Costa, Universidade Presbiteriana Mackenzie, São Paulo - Brasil \\ José Renato Campanelli, Universidade Presbiteriana Mackenzie, São Paulo - Brasil \\ Denise Elena Grillo, Universidade Presbiteriana Mackenzie, São Paulo - Brasil \\ Maria Luiza Miranda, Universidade São Judas Tadeu, São Paulo - Brasil
}

\section{RESUMO}

O objetivo do estudo foi avaliar os motivos de adesão ao grupo de ginástica geral da Universidade Presbiteriana Mackenzie, a composição corporal e as capacidades físicas dos seus integrantes. Participaram 10 indivíduos, com idade média de 22,3 anos. Para a detecção dos motivos foi utilizado um questionário semi estruturado. As características físicas foram obtidas por meio de medidas antropométricas, além de testes neuromusculares de agilidade, flexibilidade e impulsão vertical, para avaliar o nível de atividade física foi utilizado o questionário internacional de atividade física (IPAQ, 2005) versão curta e, ainda, foi feito um estudo sobre a imagem corporal. Observamos, que os motivos para a aderência ao grupo de ginástica foram: vivência diferenciada de atividades, influência dos amigos, auto superação e o fato de o indivíduo gostar de ginástica. Essa observação foi condizente com o valor do ipaq e a avaliação da imagem corporal. Concluímos que o grupo, se apresenta bem condicionado e com a composição corporal adequada às suas expectativas, portanto a GG é uma atividade prazerosa de adesão livre e espontânea.

Palavras Chave: Ginástica; Aptidão física; Adesão.

\section{GENERAL GYMNASTIC GROUP OF THE UNIVERSITY PRESBYTERIAN MACKENZIE: PHYSICAL CARACTERISTCS AND REAZONS TO SUBMIT}

\begin{abstract}
The research aims to identify the reasons for joining the Mackenzie Presbyterian University general gymnastics group, and also evaluate the body composition and physical skills of its members. There were 10 individuals with an average age of 22.3 years. In order to detect the reasons for joining the group, it was used a semi structured questionnaire with open and closed questions. The physical characteristics were obtained by anthropometric measurements of weight, height and body fat percentage, and also neuromuscular tests of agility, flexibility and vertical jump. To evaluate the level of physical activity, it was used a short version the International Physical Activity Questionnaire (IPAQ, 2005) as well as a study concerning body image. It could be observed in the results that the reasons for joining the group were: different activities experience, friendship influence, self-overcoming, and especially the exercise
\end{abstract}


attractiveness. This observation was consistent with the value of the IPAQ and the assessment of body image. As a conclusion, the group, even in the beginning of its activities, presented itself well-conditioned and with appropriate body composition, as they expected. Therefore, the GG can be a pleasant activity, which can be spontaneously joined by anyone.

Key-Words: Gymnastic; Physical fitness; Joining.

\section{GRUPO DE GIMNASIA GENERAL DE LA UNIVERSIDAD PRESBITERIANA MACKENZIE: CARACTERÍSTICA FÍSICAS Y RAZONES DE LA ADHESION}

\section{RESUMEN}

El objetivo de este estudio fue evaluar las razones de la adhesión del grupo de Gimnasia General de la Universidad Presbiteriana Mackenzie, la composición corporal, y las habilidades físicas de sus integrantes. Participaron 10 sujetos con una edad media de 22,3 años. Para la detección de las razones se utilizó un cuestionario semi-estructurado. Las características físicas se obtuvieron por medio de mediciones antropométricas y las pruebas neuromusculares de la agilidad, flexibilidad y salto vertical. Para evaluar el nivel de actividad física se utilizó el Cuestionario Internacional de Actividad Física (IPAQ, 2005), versión corta y, también, se hace un estudio sobre la imagen corporal. Vemos que las razones para el cumplimiento al grupo de gimnasia fueron: experiencia en las diferentes actividades, influencia de los compañeros, la mejora de uno mismo, y sobre todo el hecho de uno gustar de gimnasia. Esta observación era compatible con el valor del IPAQ y la evaluación de la imagen corporal. Llegamos a la conclusión de que el grupo, presenta buenas condiciones físicas y con la composición del cuerpo adecuada a sus expectativas. Por lo tanto, el GG es una actividad encantadora de libre y espontánea adhesión.

Palabras-Clave: Gimnasia; Actividad física; Adhesión.

\section{INTRODUÇÃO}


Por volta de 1940 o instituto Presbiteriano Mackenzie iniciou suas atividades em Ginástica para Todos, denominada Ginástica Rítmica Feminina tendo seu nome alterado mais tarde para Ginástica Geral (GG). As séries eram elaboradas às mãos livres ou com elementos como bola, arco, fitas, bastões, bandeiras, etc. Estas atividades eram executadas ao som de um instrumento de percussão ou de um piano e o músico adequava os tons do instrumento a serie de Ginástica já criada. A partir de então, a ginástica vem adequando suas formas de apresentação rendendo-se aos mais frenéticos e modernos ritmos que colaboram para sua evolução, no que diz respeito à criação das sérias e à criatividade de quem as elabora.

O Grupo de Ginástica Geral Mackenzie (GG MACK) foi criado em março de 2003 e estende suas atividades até o presente momento. Hoje é constituído por 35 alunos e estes do $1^{\circ}$ semestre do curso de Educação Física e alunos já formados.

A criação deste Grupo segue as orientações da Confederação Brasileira de Ginástica (CBG) entre elas, oportunizar a participação de um grande número de alunos em atividades físicas de lazer, fundamentadas nas atividades gímnicas. O grupo ainda teve como objetivo a difusão das atividades de Ginástica perante comunidades e projetos de extensão da Universidade.

O Grupo de GG Mackenzie integra atividades de ginástica acrobática, artística, rítmica, dança, artes marciais, rítmicas criativas e capoeira na elaboração de composições de GG.

Os objetivos específicos do Grupo são: propiciar aos estudantes do Curso de Educação Física participação em Atividades Rítmicas de Ginástica Geral para que possam disseminá-la através de apresentações em comemorações em eventos nacionais.

O Grupo propõe encontros semanais com intuito de criação de rotinas e ensaios para apresentações no próprio Mackenzie e/ou em eventos como fóruns, simpósios, congressos relacionadas à área da GG., bem como elaboração e apresentações de trabalhos de pesquisa em GG.

\section{REVISÃO DA LITERATURA}


Ginástica Geral (GG) é uma modalidade bastante abrangente que, fundamentada nas atividades ginásticas, utiliza-se de vários tipos de manifestações, tais como danças, expressões folclóricas e jogos, expressos através de atividades livres e criativas, objetiva promover o lazer saudável, proporcionando bem estar físico, psíquico e social aos praticantes, favorecendo inclusive a performance coletiva. ${ }^{1}$

A Ginástica Geral (GG) tem o objetivo de favorecer a participação de um grande número de pessoas e todos podem participar independente da idade, sexo ou habilidade física. Sendo assim, esta prática corporal pretende desenvolver o ser humano em suas mais diversas possibilidades.

Muitas são as historias construídas em diferentes tempos e culturas, mas nos interessa ressaltar aquela que nos ajudará na compreensão desse fenômeno, que aos poucos vem ganhando mais força no cenário nacional, aumentando o numero de aficionados. ${ }^{2}$

Sendo assim a GG é uma modalidades atual que vem recebendo adeptos a cada dia e instigando, sobretudo estudos e pesquisa. ${ }^{3}$

Nesta perspectiva podemos observar variáveis como a melhora das capacidades físicas, uma vez que estas fazem parte da maioria dos movimentos que são trabalhados de maneira contínua na prática.

Portanto a GG é uma prática que exige um conhecimento técnico de determinados movimentos, movimentos estes que atendam possibilidades de estética ao serem apresentados e que necessitam treinamento para sua execução. ${ }^{4}$ Observamos, pois que a agilidade, a resistência, a flexibilidade e a força estão entre as principais capacidades que a ginástica desenvolve, no entanto, sem apresentar o objetivo da performance, uma vez que a GG não tem como finalidade a competição, mas sim a superação através das vivências.

Apontamos assim que na atualidade a sociedade vem sendo acometida de diversos fatores de risco a saúde, e tais fatores vem sendo associado a baixos níveis de atividade física, aptidão aeróbia, e composição corporal inadequada. ${ }^{5}$

Portanto corroborando com a hipótese deste estudo, a condição corporal é subsidio para a avaliação das condições físicas, e de saúde individual ou de populações. ${ }^{6}$ 
Além desse aspecto de saúde, fica o aspecto motivacional, relevante na adesão do indivíduo à atividade. O praticante de ginástica geral procura a modalidade consciente de que não envolve competição, nem tampouco regras ou rotinas de treinamento obrigatórias, tendo sua participação na GG apoiada, principalmente, em sua motivação intrínseca. Ao mesmo tempo, podemos imaginar que existe uma ligação entre a estética corporal, tanto do praticante, quanto da própria ginástica, e a prática da modalidade sem, necessariamente, ser a principal motivação. Parece que a preocupação estética e com as capacidades físicas, em algum momento, deve ser prioridade na motivação dos participantes.

Essa característica não competitiva da modalidade leva ao questionamento da influência da participação em uma competição na adesão e na dedicação a prática da GG. Considerando que não como meta a competição, que motivos levam a adesão e que compatibilidade existe entre as capacidades físicas e a composição corporal com as solicitações da modalidade?

É possível dizer que de alguma maneira, participantes de um grupo de ginástica, apesar da não competitividade, busquem melhorar sua técnica corporal pessoal. Não podemos comparar evidentemente com as atividades competitivas, porém percebem a necessidade de treino para uma melhor execução, buscando definição e leveza nos movimentos.

\section{OBJETIVO}

Assim, o objetivo do trabalho foi avaliar os motivos de adesão, a composição corporal e as capacidades físicas do grupo de Ginástica Geral da Universidade Presbiteriana Mackenzie no início do seu ciclo anual de atividades.

\section{Objetivos Específicos}

1. Avaliar os motivos de adesão ao grupo de GG;

2. Avaliar a composição corporal através do método de dobras, a imagem corporal e o nível de atividade física;

3. Avaliar as capacidades físicas de força de Membros Inferiores (MMII, Salto horizontal e vertical), força abdominal (teste de 1 minuto), flexibilidade (banco de Wells), agilidade (Shuttle Run).

\section{MÉTODO}

Conexões: revista da Faculdade de Educação Física da UNICAMP, Campinas, v. 10, n. Especial, p. 179-191, dez. 2012. 


\section{Modalidade da Pesquisa e Local de Estudo}

Este trabalho caracterizou-se como uma pesquisa descritiva, proposto por Thomas e Nelson, ${ }^{7}$ que se propôs a fazer um levantamento da composição corporal e capacidades físicas bem como os motivos de adesão à prática da ginástica de um grupo de GG de uma Universidade da Cidade de Barueri, São Paulo, Capital, cujos integrantes são todos alunos do curso de Educação Física.

\section{Amostra}

A amostra foi composta por 10 sujeitos de ambos os sexos com média de idade de 22,3 anos.

\section{Instrumentos}

Os motivos de aderência foram detectados com um questionário semi estruturado contendo perguntas abertas e fechadas, elaborado pelos pesquisadores.

Para coleta das dobras cutâneas utilizamos o protocolo de Jackson e Pollock ${ }^{8}$ de sete dobras atendendo a necessidade de avaliar a composição corporal.

Para determinação do perfil antropométrico foram calculados o Índice de Massa Corporal (IMC - kg/m²) proposto por Queiroga, ${ }^{9}$ sendo que Organização Mundial de Saúde ${ }^{10}$ recomenda o uso destas variáveis antropométricas para a prevenção dos fatores de risco das doenças crônicas.

As capacidades físicas foram coletadas utilizando as indicações propostas por Matsudo, ${ }^{11}$ seguindo os protocolos dos testes estabelecidos pelo autor.

Para avaliar o nível de atividade física foi utilizado o Questionário Internacional de Atividade Física $^{12}$ (IPAQ) versão Curta. O IPAQ na versão curta, segundo Benedetti et al. ${ }^{13}$ se apresenta com 7 questões abertas sobre a realização de atividades físicas de intensidade leve, moderada e/ou vigorosa durante a semana em diferentes dimensões (exemplo: a caminhada como forma de trabalho ou de transporte, tarefas domésticas e de lazer, o tempo despendido com essas atividades físicas e o tempo de inatividade física). Para Hallal et al. ${ }^{14}$

O Brasil é um dos países incluídos na elaboração e validação do IPAQ, sendo este um questionário para coletar dados comparáveis de atividade física em diferentes contextos.

Em relação à imagem corporal utilizamos o instrumento Silhouette Matching Task (SMT) ou Teste para avaliação da imagem corporal proposto e adaptado por Marsh e Roche. ${ }^{15}$ 


\section{Materiais Utilizados para Coleta dos Dados}

Os instrumentos utilizados nessa pesquisa foram trena, cronometro, fita métrica, 02 blocos de madeira nas medidas determinadas e pré estabelecidas para o teste de shutle run e banco de Wells. Para o teste de impulsão vertical utilizamos a plataforma Jump System Pro, maca CEFISE, e impulsão horizontal somente a trena com demarcação da metragem horizontal.

\section{Tratamento Estatístico}

Para o tratamento estatístico foi utilizado a estatística descritiva com os resultados expressos em média do grupo. Foram, ainda, registradas as frequências relativas dos sujeitos quando a pergunta assim exigia. Os demais dados de cunho qualitativo foram apreciados a luz da análise de conteúdo de Bardin ${ }^{16}$ buscando eleger trechos significativos nas respostas dos indivíduos e categorizá-las.

A utilização dos dados dos sujeitos selecionados só foi realizada mediante a autorização dos mesmos, após assinatura da Carta de Informação ao Sujeito de Pesquisa e do Termo de Consentimento Livre e Esclarecido, ressaltando que as identidades seriam preservadas durante toda a utilização dos dados, e a liberdade dos sujeitos para abandonar a pesquisa, caso julgassem oportuno.

\section{RESULTADOS E DISCUSSÃO}

De acordo com os dados coletados no questionário contendo questões abertas e fechadas, pudemos verificar as razões que levaram os integrantes a participar do grupo de GG do Mackenzie. Os motivos indicados giram em torno da procura por uma vivência diferente, busca de horas complementares, influência dos amigos, busca por autosuperação, viajar com o grupo, mas a resposta que mais apareceu foi gostar de ginástica.

Na questão que buscou esclarecer o que o grupo de GG pode melhorar no seu dia a dia, os participantes apresentaram respostas cujos temas principais envolviam melhorar a percepção do seu próprio corpo e sua postura, trabalho amplo dos movimentos corporais, e aprendizado no sentido de observar como a ginástica pode auxiliar nos movimentos do cotidiano. Apenas um sujeito esclarece que o ritmo pode ser melhorado. 
Quando perguntamos quais os objetivos desenvolvidos pela GG, os sujeitos da pesquisa assinalaram os seguintes itens, observe o Gráfico 1:

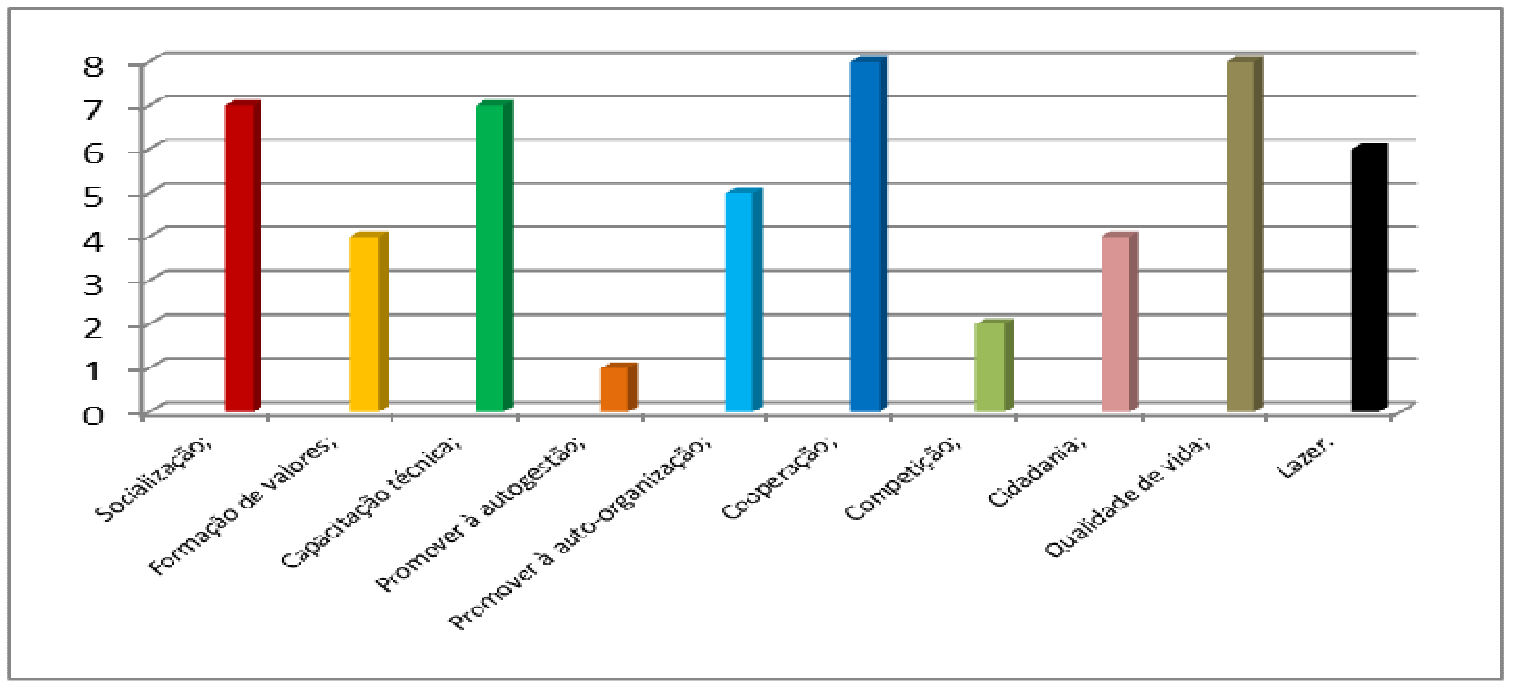

Gráfico 1: Citação dos objetivos descritos pelos sujeitos referente a GG.

Como podemos observar no gráfico, os objetivos levantados pelos integrantes do grupo de GG são socialização, capacitação técnica dos movimentos, cooperação, e qualidade de vida, objetivos estes que vêm ao encontro da proposta de desenvolvimento da Ginástica Geral, como confirman Santana e Innocente, ${ }^{17}$ quando relatam que a GG é uma modalidade de ginástica caracterizada pela "ginástica para todos", ou seja, todo e qualquer indivíduo pode praticá-la, indiferente da idade, nível social, raça, sexo, condição física ou técnica.

Confrontando nossos resultados com Santana e Innocente ${ }^{17}$ em pesquisa realizada com 17 sujeitos, verificamos que 35,29\% responderam ampliação dos conhecimentos; 29,41\% vivências teóricas, práticas e pedagógicas; $29,41 \%$ vivência de atividades diferentes; 29,41\% consciência corporal; $29,41 \%$ aspectos de confiança e superação; 23,52\% desenvolvimento do repertório motor; 17,64\% temas culturais (dança, folclore e lutas); 17,64\% trabalhar com crianças e terceira idade; $11,76 \%$ ampliação das áreas de trabalho; 11,76\% oportunidade de trabalho em escolas; $11,76 \%$ aspectos de criatividade, $11,76 \%$ relacionamento em grupo e $11,76 \%$ aspectos de lazer e diversão.

Essa informação nos sugere que o grupo está consciente do papel e das características da GG, e que esse grupo está inserido em um contexto atualizado, sendo suas observações semelhantes às descritas por Santana e Innocente. ${ }^{17}$ Essa característica é compreensível pela experiência do Conexões: revista da Faculdade de Educação Física da UNICAMP, Campinas, v. 10, n. Especial, p. 179-191, dez. 2012.186 ISSN: 1983-9030 
grupo (9 anos de existência) e também por esse grupo ser não apenas um grupo de ginástica na prática, mas também por ser um grupo de estudo sobre o tema, sem ter o foco exculsivo na prática da modalidade. Um grupo que tem buscado, em seus encontros, melhorar suas habilidades como correr, saltar ou saltitar, realizando estes movimentos básicos com expressão corporal, rítmo e sincronia.

A fim de ter uma referência do que os integrantes do grupo de GG acreditam desenvolver em relação às capacidades físicas. Esse ponto foi uma das perguntas do questionário passado ao grupo. As respostas obtidas mostram que o grupo considera a capacidade física velocidade como a menos importante (GRÁFICO 2), sendo as capacidades coordenação motora e flexibilidade consideradas as mais importantes.

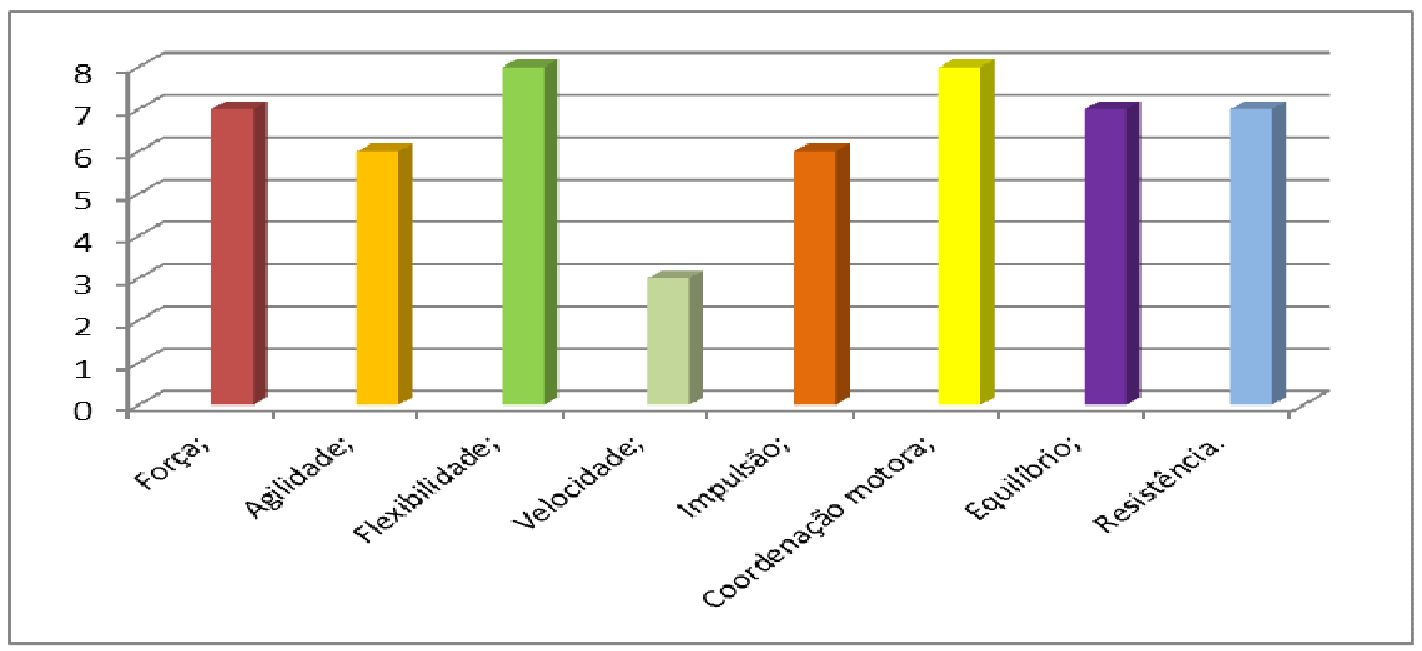

Gráfico 2 : Capacidades físicas desenvolvidas pela GG de acordo com os Sujeitos.

No entanto, quando avaliamos as capacidades físicas, atendendo a um dos objetivos do estudo, pudemos observar que o grupo se apresenta apto fisicamente, com um bom desempenho nos testes físicos, mesmo sendo o início do ciclo de atividades (GRÁFICO 3). Essa característica do grupo está associada ao nível de atividades físicas realizadas espontaneamente, em outras atividades, pelos membros do grupo.

Esse nível de condicionamento apresentado facilita a condução dos trabalhos do grupo, em termos de elaboração e apresentação de uma coreografia, mas limita a evolução, em termos de aptidão física, dos indivíduos, promovida exclusivamente pela ginástica geral. A técnica na GG vem sendo analisada cada vez mais e as mesmas são acompanhadas de melhor aptidão física, Conexões: revista da Faculdade de Educação Física da UNICAMP, Campinas, v. 10, n. Especial, p. 179-191, dez. 2012.187 ISSN: 1983-9030 
contudo não se pode exigir ou excluir, mas tentar transformar a visão dos participantes do grupo para que busquem juntos um melhor condicionamento físico. ${ }^{4}$

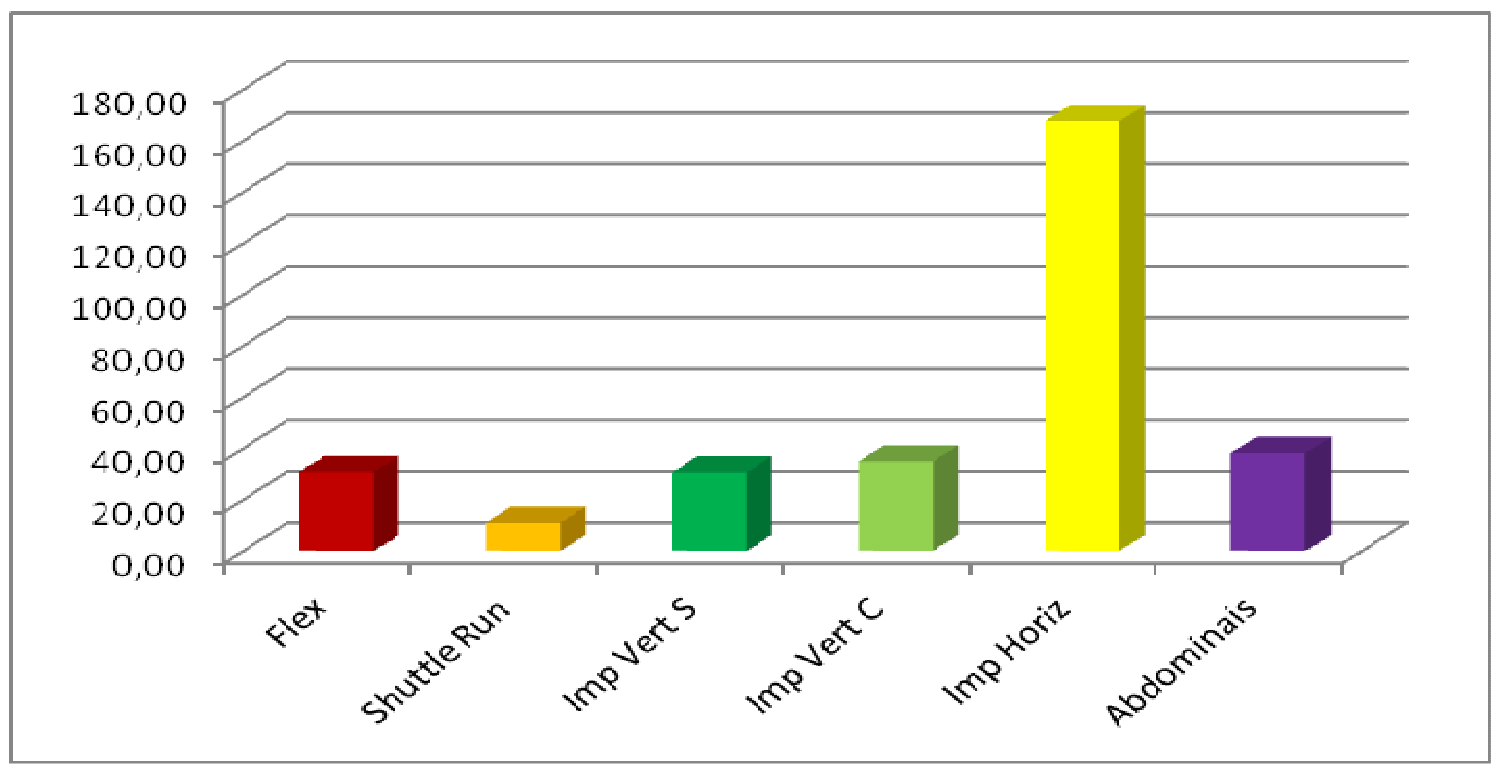

Gráfico 3: Resultados dos testes de capacidades físicas dos integrantes do Grupo de GG Mackenzie

Por fim quando perguntado aos integrantes do Grupo se eles acreditam se a GG pode ajudar na melhoria de sua composição corporal, $77,7 \%$ acreditam que sim, justificando que ajuda na definição dos músculos, ou seja, uma atividade que trabalha todos os grupos musculares, exigindo dos praticantes movimentos intensos e boa resistência aeróbia, ajudando nos movimentos do cotidiano; os outros $22,3 \%$ acreditam que não, pois, para isso, deveria ser elevado a frequência da prática (GRÁFICO 4).

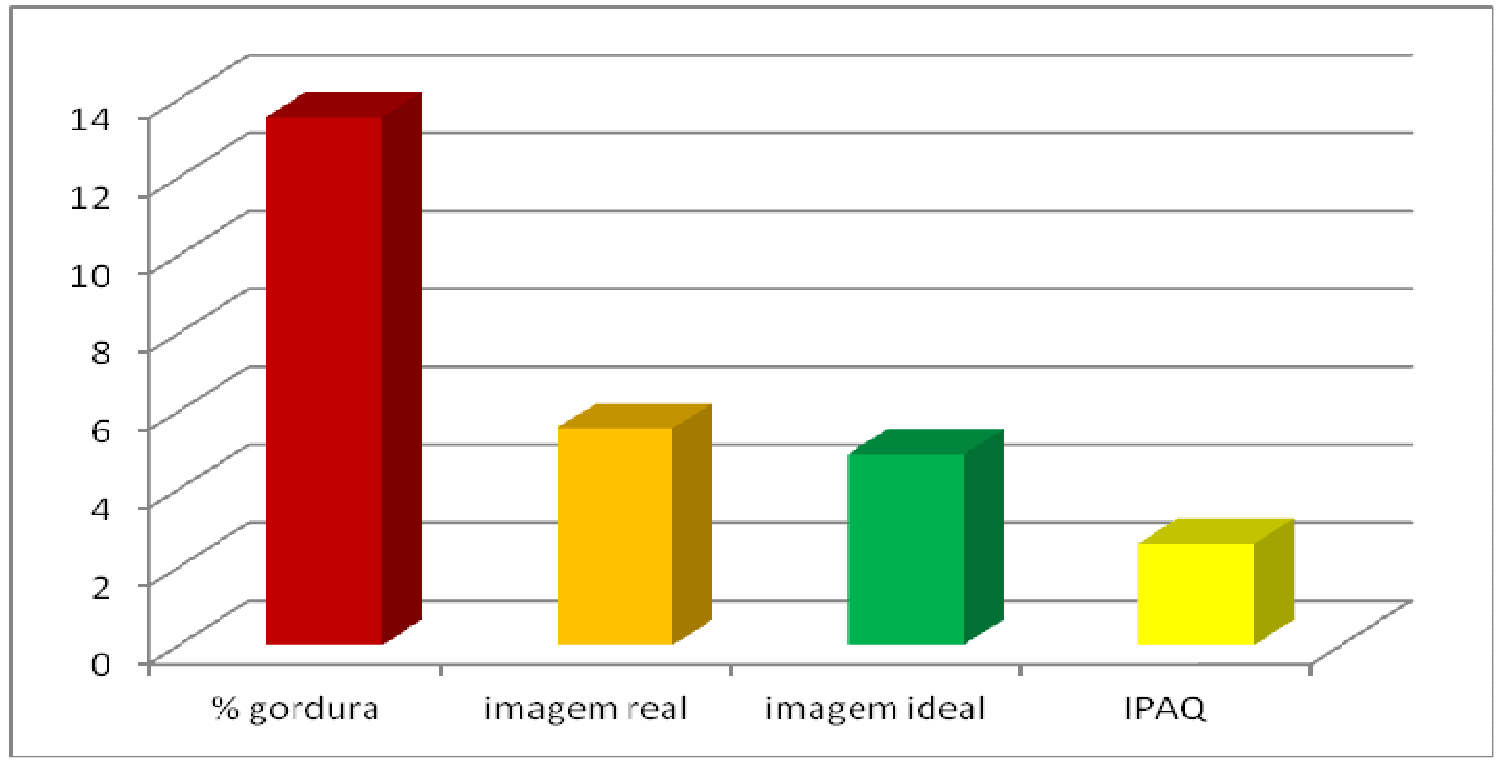

Conexões: revista da Faculdade de Educação Física da UNICAMP, Campinas, v. 10, n. Especial, p. 179-191, dez. 2012. ISSN: 1983-9030 
Gráfico 4: Resultados da avaliação da composição corporal, da imagem corporal e do nível de atividade física dos integrantes do Grupo de GG Mackenzie.

A avaliação (apresentada no GRÁFICO 4) do nível de atividade física mostrou que os indivíduos consideravam ter um nível de atividade de moderado a alto, resultando em uma pequena diferença entre a imagem corporal real e a ideal. Esses dados também são condizentes com o percentual de gordura, que se apresentou reduzido no grupo, em conformidade com o nível de atividade física medido pelo Ipaq.

\section{CONCLUSÃO}

Pudemos concluir que o grupo de ginástica geral do Mackenzie tem como principal motivo de adesão à prática o gosto pela mesma. $\mathrm{O}$ grupo, mesmo em início de suas atividades se apresenta bem condicionado e com a composição corporal adequada a suas expectativas, como mostrado pela avaliação da imagem corporal.

Ainda assim, a ideia de que a prática da ginástica pode melhorar as capacidades físicas e a composição corporal dos indivíduos é uma expectativa comum aos membros do grupo.

A GG para todos pode ser uma estratégia prazerosa que tem adesão de seus praticantes por livre e espontânea vontade, elementos que podem ser fundamentais na melhora das capacidades físicas e de composição corporal de seus praticantes.

\section{REFERÊNCIAS}

1CONFEDERAÇÃO BRASILEIRA DE GINÁSTICA (CBG). Disponível em: <http:// www.cbginastica.com.br. Acesso em: 12 abr. 2012.

²AMBrosio, M. V. B.; AMBRÓSIO, M. P. A ginástica no estado de Minas Gerais, construindo caminhos: do pioneirismo à institucionalização. Revista Mineira de Educação Física, Viçosa, n. 1, 2012.

${ }^{3}$ SOARES, C. L. Prefácio. In: PAOLIELLO, E. (Org.). Ginástica geral: experiências e reflexões. São Paulo: Phorte, 2008. 
${ }^{4}$ BORTOLETO M. A. C. Uma reflexão sobre o conceito de técnica na ginástica geral. In: PAOLIELLO, E. (Org.). Ginástica geral: experiências e reflexões. São Paulo: Phorte, 2008.

5 NASCIMENTO, R. L.; SOUZA, R. A. C.; ALMEIDA, C. M. M.; MOREIRA, S. R.. Composição corporal, mas não aptidão aeróbia e nivel de atividade física, se associa com reatividade vascular de indivíduos saudáveis. Revista Brasileira de Ciência e Movimento, Brasília, v. 19, n. 4, 2011.

${ }^{6}$ GONÇALVES, A. C.; VILLAS BOAS, R. S.; RODRIGUES, B. S. Estudo correlacional de dois protocolos duplamente indiretos para estimar composição corporal. Revista Brasileira de Ciência e Movimento, Brasília, v. 19, n. 4, 2011.

7 THOMAS, J. R.; NELSON, J. K. Métodos de pesquisa em atividade física. Porto Alegre: Artmed, 2002.

${ }^{8}$ JACKSON, A. S.; POLLOCK, M. L. Generalized equations for predicting body density of men. British Journal of Nutrition, Cambridge, v. 40, p. 497-504, 1978.

${ }^{9}$ QUEIROGA, M. R. Testes e medidas para avaliação da aptidão física relacionada à saúde em adultos. Rio de Janeiro: Guanabara Koogan, 2005.

${ }^{10}$ WORLD HEALTH ORGANIZATION (WHO). Physical status: the use and interpretation of anthropometry: report of a WHO Expert Commitee. Geneva, 1995.

11 MATSUDO V. K. R. Testes em ciências do esporte. 7. ed. São Caetano do Sul: CELAFISCS, 2005.

12 GUIDELINES for Data Processing and Analysis of the International Physical Activity Questionnaire (IPAQ): short and long forms, 2005. Disponível em: <http://www.ipaq.ki.se/scoring.pdf>. Acesso em: 11 mar. 2011.

13 BENEDETTI, T. B.; MAZO, G. Z.; BARROS, M. V. G. Aplicação do questionário internacional de atividades físicas para avaliação do nível de atividades físicas de mulheres Conexões: revista da Faculdade de Educação Física da UNICAMP, Campinas, v. 10, n. Especial, p. 179-191, dez. 2012.190 ISSN: 1983-9030 
idosas: validade concorrente e reprodutibilidade teste-reteste. Revista Brasileira de Ciência e Movimento, Brasília, v. 12, n. 1, p. 25-34, 2004.

${ }^{14}$ HALLAL P.C. et al. Evolução da pesquisa epidemiológica em atividade física no Brasil: revisão sistemática. Revista de Saúde Pública, São Paulo, v. 41, n. 3, p. 453-460, 2007.

15 MARSH, H. W.; ROCHE, L. A. Predicting self-esteem from perceptions of actual and ideal ratings of body fatness: is there only one ideal "supermodel". Research Quarterly for Exercise and Sport, Reston v. 67, n. 1, p.13-23, 1996.

${ }^{16}$ BARDIN, L. Análise de conteúdo. Lisboa: Edições 70, 2007.

${ }^{17}$ SANTANA, D. J.; INNOCENTE, L. R. Ginástica geral e o profissional de Educação Física: contribuições do projeto GG - ESEF. Pulsar, Porto Alegre, v. 3, n. 2, 2011. 